\title{
Als Kind Krebs - später magen-/darmkrank
}

Erwachsene, die sich als Kind einer Krebstherapie unterziehen mussten, haben ein erhöhtes Risiko für gastrointestinale Komplikationen. US-amerikanische Forscher untersuchten die Daten von 14358 Teilnehmern der Childhood Cancer Survivor Study. In der Studie wurde die Entwicklung von Kindern nachverfolgt, die zwischen 1976 und 1986 an Krebs erkrankt waren. Bei den Überlebenden war das Risiko für erosive Gastritiden, Verdauungsstörungen, GERD, Übelkeit,
Erbrechen, Leberzirrhose, Gallensteine, Gelbsucht, Kolitis, Verstopfung, Durchfall, Darmpolypen und Darmverschluss im späteren Leben im Vergleich zu Gesunden deutlich erhöht. Für die Schwere der Komplikationen spielten das Alter bei Diagnose der Krebserkrankung (je älter desto schlechter), die Intensität der Radio- bzw. Chemotherapie und die Schwere chirurgischer Eingriffe eine Rolle.

42. Annual Meeting of the International Society of Paediatric Oncology, Boston October 2010.

\section{HAUSHALTSPRODUKTE}

\section{Gefährliche Wohlgerüche}

„Frühlingsduft“ verspricht das Waschmittel, „Zitronenfrische“ das Geschirrspülmittel. Kaum eine Seife oder Shampoo kommt ohne besondere Duftnote aus. Was da in die Nase steigt, ist aber bedenklich, fanden Forscher der University of Washington. Sie untersuchten 25 parfümierte Haushaltsprodukte und fanden nicht weniger als 133 verschiedene

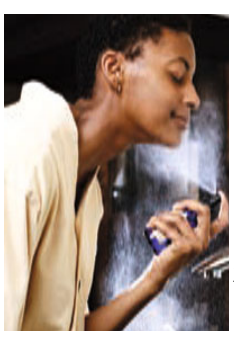

Chemikalien, die von diesen Produkten in die Luft abgegeben wurden. Etwa ein Viertel der Emissionsstoffe gilt als potenziell toxisch oder steht unter dem Verdacht, karzinogen zu sein. Produkte, die als „natürlich“ oder „organisch“ vermarktet werden, machten hierbei keine Ausnahme.

Env. Impact Asses. Rev. 2010; DOI:10.1016/ j.eiar.2010.08.002

\section{LEBENSSTIL UND KOLONKARZINOM}

\section{Jeder vierte Darmkrebsfall vermeidbar}

Eine gesunde Lebensweise kann das Darmkrebsrisiko erheblich senken. In einer prospektiven Kohortenstudie in Dänemark waren 55487 Personen zu ihren Lebensgewohnheiten befragt worden. Im Verlauf der folgenden zehn Jahre erkrankten 678 Teilnehmer an Darmkrebs. Das Risiko hing deutlich von fünf Faktoren ab, die in den Empfehlungen der WHO und des World Cancer Research Fund in einem 5-PunkteIndex zusammengefasst sind:

- Rauchverzicht

- Mindestens zo Min. Bewegung am Tag
- Beschränkung des Alkoholkonsums (weniger als 7 Drinks pro Woche für Frauen und weniger als 14 für Männer)

- Bauchumfang (unter $88 \mathrm{~cm}$ für Frauen, unter $102 \mathrm{~cm}$ für Männer)

- Gesunde, ballaststoffreiche Ernährung.

Die Autoren errechneten einen Rückgang des Krebsrisikos um 11\% pro Punkt auf der Lebensstil-Skala. Würden alle fünf Empfehlungen konsequent berücksichtigt, könnten sogar 23\% aller Darmkrebsfälle verhindert werden.

BMJ 2010;341:c5504 doi:10.1136/bmj.c5504

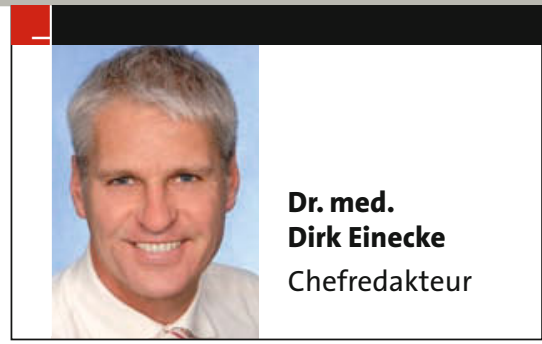

ARBEITSMEDIZIN

\section{Wer schlecht schläft, ist länger krank}

Den Zusammenhang zwischen Schlafqualität und krankheitsbedingten Fehlzeiten am Arbeitsplatz untersuchte das Finnish Institute of Occupational Health. Über 56000 Angestellte im öffentlichen Dienst wurden nach ihrer Schlafqualität gefragt und über drei Jahre beobachtet. $48 \%$ litten an Schlafstörungen. Schlechter Schlaf ging mit einem erhöhten Risiko einher, an Erkrankungen des Bewegungsapparates oder an psychischen Störungen zu erkranken. Schwere Schlafstörungen waren zusätzlich mit kardiovaskulären oder neurologischen Erkrankungen sowie mit Unfällen assoziiert. Menschen mit Schlafproblemen kehrten bei Erkrankung später an den Arbeitsplatz zurück als solche ohne.

Sleep 2010;33(10):1323-1331

\section{KOGNITIVE STÖRUNGEN Demenz nach Sepsis}

Eine Sepsis erhöht bei alten Menschen das Risiko für kognitive Schäden, ergab eine prospektive Studie vom Stony Brook University Medical Center mit Patienten, die eine schwere Sepsis überlebt hatten. Vor der Sepsis hatten 6\% der im Schnitt 77 Jahre alten Teilnehmer eine schwere kognitive Störung, nach der Klinikentlassung waren es hingegen $16,7 \%$ - eine Verdreifachung des Risikos (Odds Ratio 3,34). Viele der Patienten entwickelten nach der Sepsis auch andere bleibende Beeinträchtigungen wie Gehstörungen oder Schwierigkeiten bei täglichen Verrichtungen.

JAMA 2010;304(16):1787-1794. 